\title{
Global Existence of Solutions of the Gierer-Meinhardt System with Mixed Boundary Conditions
}

\author{
Kwadwo Antwi-Fordjour1, Marius Nkashama² \\ ${ }^{1}$ Department of Mathematics, Earlham College, Richmond, IN, USA \\ ${ }^{2}$ Department of Mathematics, University of Alabama at Birmingham, Birmingham, AL, USA \\ Email: antwikw@earlham.edu,nkashama@uab.edu
}

How to cite this paper: Antwi-Fordjour, K. and Nkashama, M. (2017) Global Existence of Solutions of the Gierer-Meinhardt System with Mixed Boundary Conditions. Applied Mathematics, 8, 857-867. https://doi.org/10.4236/am.2017.86067

Received: April 13, 2017

Accepted: June 26, 2017

Published: June 29, 2017

Copyright @ 2017 by authors and Scientific Research Publishing Inc. This work is licensed under the Creative Commons Attribution International License (CC BY 4.0).

http://creativecommons.org/licenses/by/4.0/

\section{c) (†) Open Access}

\begin{abstract}
We study the global (in time) existence of nonnegative solutions of the Gierer-Meinhardt system with mixed boundary conditions. In the research, the Robin boundary and Neumann boundary conditions were used on the activator and the inhibitor conditions respectively. Based on the priori estimates of solutions, the considerable results were obtained.
\end{abstract}

\section{Keywords}

Activator-Inhibitor System, Gierer-Meinhardt System, Robin and Neumann Boundary Conditions, Global Existence

\section{Introduction}

Biological spatial pattern formation is one area in applied mathematics undergoing vivid investigations in recent years. Most models involved in biological phenomena are of the general reaction-diffusion type considered by Turing [1]. The distinctive attribute of Turing's approach was the role of autocatalysis in coexistence with lateral inhibition. These studies led to the assumption of the existence of two chemical substances known as the activator and the inhibitor [2] [3].

One of the famous studied models in biological spatial pattern formation is the Gierer-Meinhardt system which has received numerous attention and has been extensively studied [4] [5] [6]. The Gierer-Meinhardt system was used to model the head formation of a small, fresh-water animal called hydra [4]. We consider an activator concentration $A$ and an inhibitor concentration $H$, satisfying the activator-inhibitor system given by 


$$
\begin{cases}A_{t}=\epsilon^{2} \Delta A-A+\frac{A^{p}}{H^{q}+b}, & \text { in } \Omega \times(0, T) \\ \tau H_{t}=D \Delta H-H+\frac{A^{r}}{H^{s}}, & \text { in } \Omega \times(0, T) \\ \epsilon \frac{\partial A}{\partial v}+a A=0=\frac{\partial H}{\partial v}, & \text { on } \partial \Omega \times(0, T) \\ H(x, 0)=H_{0}(x)>0, \quad A(x, 0)=A_{0}(x) \geq 0 & \text { in } \bar{\Omega}\end{cases}
$$

where $a \geq 0, b \geq 0$ and $\Omega \subset \mathbb{R}^{N}$ is a bounded smooth domain; $\Delta$ is the Laplace or diffusion operator in $\mathbb{R}^{N} ; v(x)$ is the unit outer normal at $x \in \partial \Omega$, $\partial / \partial v:=\nabla \cdot v$ is the directional derivative in the direction of the vector $v$. We assume that the reaction exponents $(p>1, q>0, r>0, s \geq 0)$ satisfy

$$
0<\frac{p-1}{r}<\frac{q}{s+1}
$$

The diffusion constants are $\epsilon>0$ and $D>0$ for the activator and inhibitor respectively. The time relaxation constant $\tau>0$ was mathematically introduced due to its usefulness on the stability of the system. The constant $b$ provides additional support to the inhibitor and may be thought of as a measure of the effectiveness of the inhibitor in suppressing the production of the activator and that of its own. In [7], the ratio in the middle of (2) is called net self-activation index, since it compares how strongly the activator activates the production of itself with how strongly it activates that of the inhibitor. On the other hand, they call the ratio on the right hand side of (2) net cross-inhibition index, since it compares how strongly the inhibitor suppresses the production of the activator with that of itself. For the the inequality in (2), we expect the production of the activator to be severely suppressed by the inhibitor.

In [4], some biological applications such as modeling of skeletal limb development, Robin boundary conditions are more realistic since the Neumann boundary conditions. A comparative numerical study of a reaction-diffusion system was made in [8] with a range of different boundary conditions and it revealed that certain types of boundary conditions selected a particular pattern modes at the expense of others. It was shown that the robustness of certain patterns could be greatly enhanced and the authors showed a possible application to skeletal pattern of limb.

Special case was considered for the Neumann boundary condition (i.e. $a=0$ ) in [5] [9]. In [5], Masuda and Takahashi proved the global solutions of the special case of (1) with $b=0$ exists for $t>0$ provided in addition to (2) one has $(p-1) / r<2 /(N+2)$, we note the strict inequality here. In [9], Jiang improved the net self-activation index noted in [5] to $(p-1) / r<1$ and showed that the solutions exists globally in time.

In this paper we consider the Robin boundary condition $(a \neq 0)$ on the activator and Neumann boundary condition on the inhibitor and study the global (in time) existence of solutions for the Gierer-Meinhardt system in (1). The theorem and lemmas in this current manuscript are inspired by [9]. We establish the global (in time) existence of (1) by proving the theorem below: 
Theorem 1. Suppose $\Omega$ is a smooth bounded domain with a smooth boundary $\partial \Omega$ in $\mathbb{R}^{N}$. Assume that $\frac{p-1}{r}<\min \left\{1, \frac{q}{s+1}\right\}$. Let $A_{0}$ and $H_{0} \in W^{2, \ell}(\Omega), \quad \ell>\max \{N, 2\}$. Then every solution $(A(x, t), H(x, t))$ of (1) exists globally in time.

\section{Proof of Theorem 1}

The local existence and uniqueness of (1) is standard and more details can be found in [10] [11]. A priori-estimates need to be ascertain in order to prove global in time existence of solutions. Let $(A, H)$ be a solution of $(1)$ in $[0, T)$. We want to ascertain that $H$ is bounded away from zero. Let

$$
u(t)=\inf _{x \in \Omega} H(x, t), \quad t \in[0, T)
$$

then

$$
u(0)=\inf _{x \in \Omega} H_{0}(x)>0
$$

Lemma 1. $u(t) \geq u(0) \mathrm{e}^{-\frac{t}{\tau}}$ for all $0 \leq t<T$.

Proof. Let

$$
H^{*}(x, t)=H(x, t)-u(0) \mathrm{e}^{-\frac{t}{\tau}}
$$

then $H^{*}(x, t)$ satisfies

$$
\begin{aligned}
H_{t}^{*} & =H_{t}-\left[u(0) \mathrm{e}^{-\frac{t}{\tau}}\right]^{\prime}=\frac{1}{\tau}\left[D \Delta H-H+\frac{A^{r}}{H^{s}}\right]+\frac{1}{\tau} u(0) \mathrm{e}^{-\frac{t}{\tau}} \\
& =\frac{1}{\tau}\left[D \Delta H^{*}-\left(H^{*}+u(0) \mathrm{e}^{-\frac{t}{\tau}}\right)+\frac{A^{r}}{H^{s}}\right]+\frac{1}{\tau} u(0) \mathrm{e}^{-\frac{t}{\tau}} \\
& =\frac{1}{\tau}\left[D \Delta H^{*}-H^{*}-u(0) \mathrm{e}^{-\frac{t}{\tau}}+\frac{A^{r}}{H^{s}}\right]+\frac{1}{\tau} u(0) \mathrm{e}^{-\frac{t}{\tau}} \\
& =\frac{1}{\tau}\left[D \Delta H^{*}-H^{*}+\frac{A^{r}}{H^{s}}\right]-\frac{1}{\tau} u(0) \mathrm{e}^{-\frac{t}{\tau}}+\frac{1}{\tau} u(0) \mathrm{e}^{-\frac{t}{\tau}} \\
& =\frac{1}{\tau}\left[D \Delta H^{*}-H^{*}+\frac{A^{r}}{H^{s}}\right]=\frac{1}{\tau}\left[D \Delta H^{*}-H^{*}\right]+\frac{A^{r}}{\tau H^{s}} \\
& \geq \frac{1}{\tau}\left[D \Delta H^{*}-H^{*}\right] \quad \text { in } \Omega \times[0, T)
\end{aligned}
$$

and

$$
\frac{\partial H^{*}}{\partial v}=\frac{\partial}{\partial v}\left[H(x, t)-u(0) \mathrm{e}^{-\frac{t}{\tau}}\right]=\frac{\partial}{\partial v} H(x, t)
$$

but

$$
\frac{\partial H}{\partial v}=0 \quad \text { on } \partial \Omega \times[0, T)
$$

thus

$$
\frac{\partial H^{*}}{\partial v}=0 \quad \text { on } \partial \Omega \times[0, T) .
$$


Additionally at $t=0$, from (3)

$$
H^{*}(x, 0)=H(x, 0)-u(0)=H(x, 0)-\inf _{x \in \Omega} H(x, 0) \geq 0
$$

So $H^{*}(x, 0) \geq 0$ for any $x \in \Omega$.

Hence from maximum principle, $H^{*}(x, t) \geq 0$ in $\bar{\Omega} \times[0, T)$ and thus

$$
\begin{gathered}
H(x, t)-u(0) \mathrm{e}^{-\frac{t}{\tau}} \geq 0, \\
u(t)=\inf _{x \in \Omega} H(x, t) \geq u(0) \mathrm{e}^{-\frac{t}{\tau}}, \quad t \in[0, T)
\end{gathered}
$$

Lemma 2. For any two constants $\alpha>1, \beta \geq 0$, let $\frac{p-1}{r}<\min \left\{1, \frac{q}{s+1}\right\}$. Define

$$
h_{\alpha, \beta}(t)=\int_{\Omega} \frac{A^{\alpha}(x, t)}{H^{\beta}(x, t)} \mathrm{d} x, \quad 0 \leq t<T
$$

Suppose

$$
2 \varepsilon \sqrt{\tau D(\alpha-1)(\beta+1)} \geq\left(\tau \epsilon^{2}+D\right) \sqrt{\alpha \beta}
$$

then

$$
\dot{h}_{\alpha, \beta}(t) \leq\left(\frac{\beta}{\tau}-\alpha\right) h_{\alpha, \beta}(t)+C u^{-\delta} h_{\alpha, \beta}^{1-\vartheta}(t)
$$

Here

$$
\vartheta=\frac{\sigma}{\alpha}\left(\frac{r}{r-(p-1)-\sigma}\right)
$$

and

$$
\delta=\frac{r}{r-(p-1)-\sigma}\left[q-\frac{(s+1)(p-1)}{r}-\left(\frac{s+1}{r}-\frac{\beta}{\alpha}\right) \sigma\right]
$$

where $\delta>0, \sigma>0, \vartheta \in(0,1)$ and

$$
C=\left[\alpha(\beta / \tau)^{-\frac{p-1+\sigma}{r}}\right]^{\frac{r}{r-(p-1)-\sigma}}|\Omega|^{\vartheta}
$$

Proof. Let $\alpha>1$ and $\beta \geq 0$,

$$
\begin{aligned}
\dot{h}_{\alpha, \beta}(t)= & \frac{\mathrm{d}}{\mathrm{d} t}\left(\int_{\Omega} \frac{A^{\alpha}}{H^{\beta}} \mathrm{d} x\right)=\int_{\Omega}\left(\frac{A^{\alpha}}{H^{\beta}}\right)_{t} \mathrm{~d} x=\int_{\Omega}\left[\alpha \frac{A^{\alpha-1}}{H^{\beta}} A_{t}-\beta \frac{A^{\alpha}}{H^{\beta+1}} H_{t}\right] \mathrm{d} x \\
= & \int_{\Omega}\left[\alpha \frac{A^{\alpha-1}}{H^{\beta}}\left(\epsilon^{2} \Delta A-A+\frac{A^{p}}{H^{q}+b}\right)-\beta \frac{A^{\alpha}}{\tau H^{\beta+1}}\left(D \Delta H-H+\frac{A^{r}}{H^{s}}\right)\right] \mathrm{d} x \\
= & \int_{\Omega} \alpha \epsilon^{2} \frac{A^{\alpha-1}}{H^{\beta}} \Delta A \mathrm{~d} x-\int_{\Omega} \alpha \frac{A^{\alpha}}{H^{\beta}} \mathrm{d} x+\int_{\Omega} \alpha \frac{A^{\alpha+p-1}}{H^{\beta}\left(H^{q}+b\right)} \mathrm{d} x \\
& -\int_{\Omega} \beta D \frac{A^{\alpha}}{\tau H^{\beta+1}} \Delta H \mathrm{~d} x+\int_{\Omega} \beta \frac{A^{\alpha}}{\tau H^{\beta}} \mathrm{d} x-\int_{\Omega} \beta \frac{A^{\alpha+r}}{\tau H^{\beta+s+1}} \mathrm{~d} x \\
= & \left(\frac{\beta}{\tau}-\alpha\right) \int_{\Omega} \frac{A^{\alpha}}{H^{\beta}} \mathrm{d} x+\alpha \epsilon^{2} \int_{\Omega} \frac{A^{\alpha-1}}{H^{\beta}} \Delta A \mathrm{~d} x-\frac{\beta D}{\tau} \int_{\Omega} \frac{A^{\alpha}}{H^{\beta+1}} \Delta H \mathrm{~d} x+\alpha \int_{\Omega} \frac{A^{\alpha+p-1}}{H^{\beta}\left(H^{q}+b\right)} \mathrm{d} x-\frac{\beta}{\tau} \int_{\Omega} \frac{A^{\alpha+r}}{H^{\beta+s+1}} \mathrm{~d} x
\end{aligned}
$$


But

$$
\begin{aligned}
\alpha \epsilon^{2} \int_{\Omega} \frac{A^{\alpha-1}}{H^{\beta}} \Delta A \mathrm{~d} x= & \alpha \epsilon^{2} \int_{\partial \Omega} \frac{A^{\alpha-1}}{H^{\beta}} \nabla A \cdot v \mathrm{~d} S-\alpha \epsilon^{2}(\alpha-1) \int_{\Omega} \frac{A^{\alpha-2}}{H^{\beta}}|\nabla A|^{2} \mathrm{~d} x \\
& +\alpha \epsilon^{2} \beta \int_{\Omega} \frac{A^{\alpha-1}}{H^{\beta+1}} \nabla A \nabla H \mathrm{~d} x \\
= & \alpha \epsilon^{2} \int_{\partial \Omega} \frac{A^{\alpha-1}}{H^{\beta}}\left(\frac{-a A}{\epsilon}\right) \mathrm{d} S-\alpha \varepsilon^{2}(\alpha-1) \int_{\Omega} \frac{A^{\alpha-2}}{H^{\beta}}|\nabla A|^{2} \mathrm{~d} x \\
& +\alpha \epsilon^{2} \beta \int_{\Omega} \frac{A^{\alpha-1}}{H^{\beta+1}} \nabla A \nabla H \mathrm{~d} x \\
= & -\alpha \epsilon a \int_{\partial \Omega} \frac{A^{\alpha}}{H^{\beta}} \mathrm{d} S-\alpha \epsilon^{2}(\alpha-1) \int_{\Omega} \frac{A^{\alpha-2}}{H^{\beta}}|\nabla A|^{2} \mathrm{~d} x \\
& +\alpha \epsilon^{2} \beta \int_{\Omega} \frac{A^{\alpha-1}}{H^{\beta+1}} \nabla A \nabla H \mathrm{~d} x
\end{aligned}
$$

Additionally,

$$
\begin{aligned}
\frac{\beta D}{\tau} \int_{\Omega} \frac{A^{\alpha}}{H^{\beta+1}} \Delta H \mathrm{~d} x= & \frac{\beta D}{\tau} \int_{\partial \Omega} \frac{A^{\alpha}}{H^{\beta+1}} \nabla H \cdot v \mathrm{~d} S+\frac{\beta(\beta+1) D}{\tau} \int_{\Omega} \frac{A^{\alpha}}{H^{\beta+2}}|\nabla H|^{2} \mathrm{~d} x \\
& -\frac{\alpha \beta D}{\tau} \int_{\Omega} \frac{A^{\alpha-1}}{H^{\beta+1}} \nabla A \nabla H \mathrm{~d} x \\
= & \frac{\beta D}{\tau} \int_{\partial \Omega} \frac{A^{\alpha}}{H^{\beta+1}}(0) \mathrm{d} S+\frac{\beta(\beta+1) D}{\tau} \int_{\Omega} \frac{A^{\alpha}}{H^{\beta+2}}|\nabla H|^{2} \mathrm{~d} x \\
& -\frac{\alpha \beta D}{\tau} \int_{\Omega} \frac{A^{\alpha-1}}{H^{\beta+1}} \nabla A \nabla H \mathrm{~d} x \\
= & \frac{\beta(\beta+1) D}{\tau} \int_{\Omega} \frac{A^{\alpha}}{H^{\beta+2}}|\nabla H|^{2} \mathrm{~d} x-\frac{\alpha \beta D}{\tau} \int_{\Omega} \frac{A^{\alpha-1}}{H^{\beta+1}} \nabla A \nabla H \mathrm{~d} x
\end{aligned}
$$

now we have,

$$
\begin{aligned}
& \dot{h}_{\alpha, \beta}(t)=\left(\frac{\beta}{\tau}-\alpha\right) \int_{\Omega} \frac{A^{\alpha}}{H^{\beta}} \mathrm{d} x-\alpha \epsilon a \int_{\partial \Omega} \frac{A^{\alpha}}{H^{\beta}} \mathrm{d} S-\alpha \epsilon^{2}(\alpha-1) \int_{\Omega} \frac{A^{\alpha-2}}{H^{\beta}}|\nabla A|^{2} \mathrm{~d} x \\
& +\alpha \beta\left(\epsilon^{2}+\frac{D}{\tau}\right) \int_{\Omega} \frac{A^{\alpha-1}}{H^{\beta+1}} \nabla A \nabla H \mathrm{~d} x-\frac{\beta(\beta+1) D}{\tau} \int_{\Omega} \frac{A^{\alpha}}{H^{\beta+2}}|\nabla H|^{2} \mathrm{~d} x \\
& +\alpha \int_{\Omega} \frac{A^{\alpha+p-1}}{H^{\beta+q}} \mathrm{~d} x-\frac{\beta}{\tau} \int_{\Omega} \frac{A^{\alpha+r}}{H^{\beta+s+1}} \mathrm{~d} x . \\
& =\left(\frac{\beta}{\tau}-\alpha\right) \int_{\Omega} \frac{A^{\alpha}}{H^{\beta}} \mathrm{d} x-\alpha \epsilon a \int_{\partial \Omega} \frac{A^{\alpha}}{H^{\beta}} \mathrm{d} S-\alpha \epsilon^{2}(\alpha-1) \int_{\Omega} \frac{A^{\alpha}}{H^{\beta}}\left|\frac{\nabla A}{A}\right|^{2} \mathrm{~d} x \\
& +\alpha \beta\left(\epsilon^{2}+\frac{D}{\tau}\right) \int_{\Omega} \frac{A^{\alpha}}{H^{\beta}} \frac{\nabla H}{H} \frac{\nabla A}{A} \mathrm{~d} x-\frac{\beta(\beta+1) D}{\tau} \int_{\Omega} \frac{A^{\alpha}}{H^{\beta}}\left|\frac{\nabla H}{H}\right|^{2} \mathrm{~d} x \\
& +\alpha \int_{\Omega} \frac{A^{\alpha+p-1}}{H^{\beta+q}} \mathrm{~d} x-\frac{\beta}{\tau} \int_{\Omega} \frac{A^{\alpha+r}}{H^{\beta+s+1}} \mathrm{~d} x . \\
& =\left(\frac{\beta}{\tau}-\alpha\right) \int_{\Omega} \frac{A^{\alpha}}{H^{\beta}} \mathrm{d} x-\alpha \epsilon a \int_{\partial \Omega} \frac{A^{\alpha}}{H^{\beta}} \mathrm{d} S+\alpha \int_{\Omega} \frac{A^{\alpha+p-1}}{H^{\beta+q}} \mathrm{~d} x-\frac{\beta}{\tau} \int_{\Omega} \frac{A^{\alpha+r}}{H^{\beta+s+1}} \mathrm{~d} x \\
& +\int_{\Omega} \frac{A^{\alpha}}{H^{\beta}}\left[-\alpha \epsilon^{2}(\alpha-1)\left|\frac{\nabla A}{A}\right|^{2} \mathrm{~d} x+\alpha \beta\left(\epsilon^{2}+\frac{D}{\tau}\right) \frac{\nabla H}{H} \frac{\nabla A}{A} \mathrm{~d} x\right. \\
& \left.-\frac{\beta(\beta+1) D}{\tau}\left|\frac{\nabla H}{H}\right|^{2} \mathrm{~d} x\right]
\end{aligned}
$$


We deduce from above a quadratic equation involving $\frac{\nabla A}{A}$ and $\frac{\nabla H}{H}$. Let us fix $\alpha>1$ and choose $\beta \geq 0$, we have

$$
2 \epsilon \sqrt{\tau D(\alpha-1)(\beta+1)} \geq\left(\tau \epsilon^{2}+D\right) \sqrt{\alpha \beta}
$$

therefore the quadratic form involving $\frac{\nabla A}{A}$ and $\frac{\nabla H}{H}$ in the inequality above is non-positive since its determinant

$$
\left[\alpha \beta\left(\epsilon^{2}+\frac{D}{\tau}\right) \frac{\nabla H}{H}\right]^{2} \leq 4\left[-\epsilon^{2} \alpha(\alpha-1)\right]\left[-\frac{\beta D}{\tau}(\beta+1)\left|\frac{\nabla H}{H}\right|^{2}\right] .
$$

Thus

$$
\begin{aligned}
\dot{h}_{\alpha, \beta}(t) & \leq\left(\frac{\beta}{\tau}-\alpha\right) \int_{\Omega} \frac{A^{\alpha}}{H^{\beta}} \mathrm{d} x-\alpha \epsilon a \int_{\partial \Omega} \frac{A^{\alpha}}{H^{\beta}} \mathrm{d} S+\alpha \int_{\Omega} \frac{A^{\alpha+p-1}}{H^{\beta+q}} \mathrm{~d} x-\frac{\beta}{\tau} \int_{\Omega} \frac{A^{\alpha+r}}{H^{\beta+s+1}} \mathrm{~d} x \\
& \leq\left(\frac{\beta}{\tau}-\alpha\right) \int_{\Omega} \frac{A^{\alpha}}{H^{\beta}} \mathrm{d} x+\alpha \int_{\Omega} \frac{A^{\alpha+p-1}}{H^{\beta+q}} \mathrm{~d} x-\frac{\beta}{\tau} \int_{\Omega} \frac{A^{\alpha+r}}{H^{\beta+s+1}} \mathrm{~d} x \\
& =\left(\frac{\beta}{\tau}-\alpha\right) h_{\alpha, \beta}(t)+\alpha \int_{\Omega} \frac{A^{\alpha+p-1}}{H^{\beta+q}} \mathrm{~d} x-\frac{\beta}{\tau} \int_{\Omega} \frac{A^{\alpha+r}}{H^{\beta+s+1}} \mathrm{~d} x .
\end{aligned}
$$

We have

$$
\frac{p-1}{r}<1 \Rightarrow r-(p-1)>0
$$

and

$$
\frac{p-1}{r}<\frac{q}{s+1} \Rightarrow q>\frac{(p-1)(s+1)}{r}
$$

we choose $\sigma>0$ sufficiently small such that

$$
r-(p-1)>\sigma
$$

and

$$
q>\frac{(p-1)(s+1)}{r}+\left(\frac{s+1}{r}-\frac{\beta}{\alpha}\right) \sigma .
$$

Now, we write

$$
\begin{aligned}
& \frac{A^{\alpha+p-1}}{H^{\beta}\left(H^{q}+b\right)} \leq \frac{A^{\alpha+p-1}}{H^{\beta} H^{q}}=\frac{A^{\alpha+p-1}}{H^{\beta} H^{\delta\left(\frac{r-(p-1)-\sigma}{r}\right)+\frac{(p-1)(s+1)}{r}+\left(\frac{s+1}{r}-\frac{\beta}{\alpha}\right) \sigma}} \\
= & \frac{A^{\alpha+p-1}}{H^{\beta} H^{\delta\left(\frac{r-(p-1)-\sigma}{r}\right)} H^{\frac{(p-1)(s+1)}{r}} H^{\left(\frac{s+1}{r}-\frac{\beta}{\alpha}\right) \sigma}}=\frac{A^{\alpha+p-1} H^{\beta \frac{\sigma}{\alpha}}}{H^{\delta\left(\frac{r-(p-1)-\sigma}{r}\right)} H^{\frac{(p-1)(s+1)}{r}} H^{\frac{s+1}{r} \sigma}} \\
= & \frac{A^{\alpha+p-1} H^{\beta \frac{\sigma}{\alpha}}}{H^{\delta} H^{\delta\left(\frac{r-(p-1)-\sigma}{r}\right)} H^{(s+1) \frac{p-1+\sigma}{r}}}=\frac{A^{\alpha+p-1} H^{\beta \frac{\sigma}{\alpha}}}{H^{\beta} H^{\delta\left(\frac{r-(p-1)-\sigma}{r}\right)} H^{(s+1) \frac{p-1+\sigma}{r}}} \\
= & \frac{A^{\alpha+p-1} H^{\beta \vartheta\left(1-\frac{p-1+\sigma}{r}\right)}}{H^{\beta-\beta \frac{p-1+\sigma}{r}+\beta \frac{p-1+\sigma}{r}} H^{\delta\left(1-\frac{p-1+\sigma}{r}\right)} H^{(s+1) \frac{p-1+\sigma}{r}}}=\frac{A^{\alpha+p-1} H^{\beta\left(1-\frac{p-1+\sigma}{r}\right)} H^{\delta\left(1-\frac{p-1+\sigma}{r}\right)} H^{(\beta+s+1) \frac{p-1+\sigma}{r}}}{\left.H^{(1-\sigma}\right)}
\end{aligned}
$$


but

$$
\begin{aligned}
A^{\alpha+p-1} & =\frac{A^{\alpha} A^{p-1} A^{\sigma}}{A^{\sigma}}=\frac{A^{\alpha} A^{p-1+\sigma}}{A^{\alpha \vartheta\left(1-\frac{p-1+\sigma}{r}\right)}} \\
& =\frac{A^{\alpha\left(1-\frac{p-1+\sigma}{r}+\frac{p-1+\sigma}{r}\right)} A^{r \frac{p-1+\sigma}{r}}}{A^{\alpha \vartheta\left(1-\frac{p-1+\sigma}{r}\right)}} \\
& =\frac{A^{\alpha\left(1-\frac{p-1+\sigma}{r}\right)} A^{\alpha \frac{p-1+\sigma}{r}} A^{r \frac{p-1+\sigma}{r}}}{A^{\alpha \vartheta\left(1-\frac{p-1+\sigma}{r}\right)}} \\
& =A^{\alpha(1-\vartheta)\left(1-\frac{p-1+\sigma}{r}\right)} A^{(\alpha+r) \frac{p-1+\sigma}{r}}
\end{aligned}
$$

thus

$$
\begin{aligned}
\frac{A^{\alpha+p-1}}{H^{\beta+q}} & =\frac{A^{\alpha(1-\vartheta)\left(1-\frac{p-1+\sigma}{r}\right)} A^{(\alpha+r) \frac{p-1+\sigma}{r}} H^{\beta \vartheta\left(1-\frac{p-1+\sigma}{r}\right)}}{H^{\beta\left(1-\frac{p-1+\sigma}{r}\right)} H^{\delta\left(1-\frac{p-1+\sigma}{r}\right)} H^{(\beta+s+1) \frac{p-1+\sigma}{r}}} \\
& =\frac{H^{-\delta\left(1-\frac{p-1+\sigma}{r}\right)} A^{\alpha(1-\vartheta)\left(1-\frac{p-1+\sigma}{r}\right)} A^{(\alpha+r) \frac{p-1+\sigma}{r}}}{H^{\beta(1-\vartheta)\left(1-\frac{p-1+\sigma}{r}\right)} H^{(\beta+s+1) \frac{p-1+\sigma}{r}}} \\
& =\left[H^{-\delta}\left(\frac{A^{\alpha}}{H^{\beta}}\right)^{1-\vartheta}\right]^{1-\frac{p-1+\sigma}{r}}\left(\frac{A^{\alpha+r}}{H^{\beta+s+1}}\right)^{\frac{p-1+\sigma}{r}}
\end{aligned}
$$

where $\vartheta$ and $\delta$ are defined by (6) and (7)

$$
\begin{aligned}
\alpha \frac{A^{\alpha+p-1}}{H^{\beta}\left(H^{q}+b\right)} & \leq \alpha\left[H^{-\delta}\left(\frac{A^{\alpha}}{H^{\beta}}\right)^{1-\vartheta}\right]^{1-\frac{p-1+\sigma}{r}}\left(\frac{A^{\alpha+r}}{H^{\beta+s+1}}\right)^{\frac{p-1+\sigma}{r}} \\
& \leq \alpha\left[u^{-\delta}\left(\frac{A^{\alpha}}{H^{\beta}}\right)^{1-\vartheta}\right]^{1-\frac{p-1+\sigma}{r}}\left(\frac{A^{\alpha+r}}{H^{\beta+s+1}}\right)^{\frac{p-1+\sigma}{r}} \\
& =\alpha\left[u^{-\delta}\left(\frac{A^{\alpha}}{H^{\beta}}\right)^{1-\vartheta}\right]^{1-\frac{p-1+\sigma}{r}}\left(\frac{\beta \tau^{-1}}{\beta \tau^{-1}} \frac{A^{\alpha+r}}{H^{\beta+s+1}}\right)^{\frac{p-1+\sigma}{r}} \\
& =\alpha\left(\frac{\beta}{\tau}\right)^{-\frac{p-1+\sigma}{r}}\left[u^{-\delta}\left(\frac{A^{\alpha}}{H^{\beta}}\right)^{1-\vartheta}\right]^{1-\frac{p-1+\sigma}{r}}\left(\frac{\beta}{\tau} \frac{A^{\alpha+r}}{H^{\beta+s+1}}\right)^{\frac{p-1+\sigma}{r}}
\end{aligned}
$$

by Young's inequality, we obtain

$$
\begin{aligned}
& \alpha \frac{A^{\alpha+p-1}}{H^{\beta}\left(H^{q}+b\right)} \leq\left[\alpha\left(\frac{\beta}{\tau}\right)^{-\frac{p-1+\sigma}{r}}\right]^{\frac{r}{r-(p-1)-\sigma}} u^{-\delta}\left(\frac{A^{\alpha}}{H^{\beta}}\right)^{1-\vartheta}+\frac{\beta}{\tau} \frac{A^{\alpha+r}}{H^{\beta+s+1}} \\
& \alpha \frac{A^{\alpha+p-1}}{H^{\beta}\left(H^{q}+b\right)}-\frac{\beta}{\tau} \frac{A^{\alpha+r}}{H^{\beta+s+1}} \leq\left[\alpha\left(\frac{\beta}{\tau}\right)^{-\frac{p-1+\sigma}{r}}\right]^{\frac{r}{r-(p-1)-\sigma}} u^{-\delta}\left(\frac{A^{\alpha}}{H^{\beta}}\right)^{1-\vartheta} .
\end{aligned}
$$


Therefore

$$
\alpha \int_{\Omega} \frac{A^{\alpha+p-1}}{H^{\beta}\left(H^{q}+b\right)} \mathrm{d} x-\frac{\beta}{\tau} \int_{\Omega} \frac{A^{\alpha+r}}{H^{\beta+s+1}} \mathrm{~d} x \leq\left[\alpha\left(\frac{\beta}{\tau}\right)^{-\frac{p-1+\sigma}{r}}\right]^{\frac{r}{r-(p-1)-\sigma}} u^{-\delta} \int_{\Omega}\left(\frac{A^{\alpha}}{H^{\beta}}\right)^{1-\vartheta} \mathrm{d} x
$$

but by Hölder's inequality

$$
\int_{\Omega}\left(\frac{A^{\alpha}}{H^{\beta}}\right)^{1-\vartheta} d x \leq\left(\int_{\Omega} \mathrm{d} x\right)^{\vartheta}\left(\int_{\Omega} \frac{A^{\alpha}}{H^{\beta}} \mathrm{d} x\right)^{1-\vartheta}=|\Omega|^{\vartheta} h_{\alpha, \beta}^{1-\vartheta} .
$$

Thus

$$
\begin{aligned}
& \alpha \int_{\Omega} \frac{A^{\alpha+p-1}}{H^{\beta}\left(H^{q}+b\right)} \mathrm{d} x-\frac{\beta}{\tau} \int_{\Omega} \frac{A^{\alpha+r}}{H^{\beta+s+1}} \mathrm{~d} x \\
& \leq\left[\alpha\left(\frac{\beta}{\tau}\right)^{-\frac{p-1+\sigma}{r}}\right]^{\frac{r}{r-(p-1)-\sigma}} u^{-\delta}|\Omega|^{\vartheta} h_{\alpha, \beta}^{1-\vartheta}=C u^{-\delta} h_{\alpha, \beta}^{1-\vartheta} .
\end{aligned}
$$

Finally,

$$
\dot{h}_{\alpha, \beta}(t) \leq\left(\frac{\beta}{\tau}-\alpha\right) h_{\alpha, \beta}+C u^{-\delta} h_{\alpha, \beta}^{1-\vartheta}
$$

Remark 1 . The condition in (4) is true for any

$$
\alpha \geq 2 \text { and } 0<\beta \leq \frac{1}{2 K}
$$

where $K \geq \max \left\{\frac{\tau \epsilon^{2}}{D}, \frac{D}{\tau \epsilon^{2}}\right\}$

Lemma 3. Let $0 \leq \delta<1, \theta>0$ and $\zeta>0$ on $(0, T)$ be an integrable function. Let $h_{\alpha, \beta}=h_{\alpha, \beta}(t)$ be a nonnegative function on $[0, T)$ satisfying the differential inequality

$$
\dot{h}_{\alpha, \beta}(t) \leq-\theta h_{\alpha, \beta}+\zeta h_{\alpha, \beta}^{\delta}, \quad 0 \leq t<T .
$$

Then

$$
h_{\alpha, \beta}(t) \leq \kappa, \quad 0 \leq t<T
$$

where $\kappa$ is the maximal root of the algebraic equation

$$
x-G(\zeta) x^{\delta}=h_{\alpha, \beta}(0) .
$$

Moreover, if $T=\infty$, we have

$$
\limsup _{t \rightarrow \infty} h_{\alpha, \beta}(t) \leq \kappa_{\infty},
$$

where $\kappa_{\infty}$ is the maximal root of the algebraic equation

$$
x-G_{\infty}(\zeta) x^{\delta}=0 .
$$

Proof.

$$
\dot{h}_{\alpha, \beta}(t) \leq-\theta h_{\alpha, \beta}+\zeta h_{\alpha, \beta}^{\delta}
$$




$$
\begin{gathered}
\dot{h}_{\alpha, \beta}(t)+\theta h_{\alpha, \beta} \leq \zeta h_{\alpha, \beta}^{\delta} \\
\mathrm{e}^{\theta t}\left[\dot{h}_{\alpha, \beta}(t)+\theta h_{\alpha, \beta}\right] \leq \mathrm{e}^{\theta t} \zeta h_{\alpha, \beta}^{\delta} \\
\frac{\mathrm{d}}{\mathrm{d} t}\left[\mathrm{e}^{\theta t} h_{\alpha, \beta}\right] \leq \zeta \mathrm{e}^{\theta t} h_{\alpha, \beta}^{\delta} \\
\int_{0}^{t} \frac{\mathrm{d}}{\mathrm{d} t}\left[\mathrm{e}^{\theta \chi} h_{\alpha, \beta}\right] \mathrm{d} \chi \leq \int_{0}^{t} \mathrm{e}^{\theta \chi} \zeta(\chi) h_{\alpha, \beta}^{\delta}(\chi) \mathrm{d} \chi \\
\mathrm{e}^{\theta t} h_{\alpha, \beta}(t)-h_{\alpha, \beta}(0) \leq \int_{0}^{t} \mathrm{e}^{\theta \chi} \zeta(\chi) h_{\alpha, \beta}^{\delta}(\chi) \mathrm{d} \chi \\
\mathrm{e}^{\theta t} h_{\alpha, \beta}(t) \leq h_{\alpha, \beta}(0)+\int_{0}^{t} \mathrm{e}^{\theta \chi} \zeta(\chi) h_{\alpha, \beta}^{\delta}(\chi) \mathrm{d} \chi \\
h_{\alpha, \beta}(t) \leq \mathrm{e}^{-\theta t} h_{\alpha, \beta}(0)+\int_{0}^{t} \mathrm{e}^{-\theta(t-\chi)} \zeta(\chi) h_{\alpha, \beta}^{\delta}(\chi) \mathrm{d} \chi .
\end{gathered}
$$

Let

$$
\overline{h_{\alpha, \beta}}(t)=\sup _{0<\chi<t} h_{\alpha, \beta}(\chi),
$$

and

$$
G(\zeta):=\sup _{0<t<T} \int_{0}^{t} \mathrm{e}^{-\theta(t-\chi)} \zeta(\chi) \mathrm{d} \chi
$$

in particular, at $T=\infty$

$$
G_{\infty}(\zeta):=\limsup _{t \rightarrow \infty} \int_{0}^{t} \mathrm{e}^{-\theta(t-\chi)} \zeta(\chi) \mathrm{d} \chi
$$

we obtain now that

$$
\overline{h_{\alpha, \beta}}(t) \leq h_{\alpha, \beta}(0)+G(\zeta) \overline{h_{\alpha, \beta}^{\delta}}(t)
$$

Notice that the quantity $G(\zeta)$ is finite and hence (9) follows from (12). As $t \rightarrow \infty$ in (11), we ascertain

$$
\limsup _{t \rightarrow \infty} h_{\alpha, \beta}(t) \leq G_{\infty}(\zeta) \limsup _{t \rightarrow \infty} h_{\alpha, \beta}^{\delta}(t)
$$

thus (10) follows since $G_{\infty}(\zeta)$ is finite.

The next Lemma follows after applying Lemma 3 to (5).

Lemma 4. For any $\alpha, \beta>0$ such that $\beta<\tau \alpha$, and all conditions in Lemma 2 hold true. Then there exists a constant $C(T)=C_{\alpha, \beta}(T) \leq \infty$ such that

$$
h_{\alpha, \beta}(t) \leq C(T)
$$

for all $t \in[0, T)$.

Proof. For sufficiently small $\beta>0$, such that $\beta<\tau \alpha$ with $\alpha>1$, we obtain

$$
2 \epsilon \sqrt{\tau D(\alpha-1)(\beta+1)} \geq\left(\tau \epsilon^{2}+D\right) \sqrt{\alpha \beta}, \quad \frac{\beta}{\tau}-\alpha<0,
$$

therefore we deduce from Lemma 2 that $h_{\alpha, \beta}$ satisfies

$$
\dot{h}_{\alpha, \beta}(t) \leq\left(\frac{\beta}{\tau}-\alpha\right) h_{\alpha, \beta}+C u^{-\delta} h_{\alpha, \beta}^{1-9} .
$$

Since

$$
\frac{\beta}{\tau}-\alpha<0
$$


and

$$
u(t) \geq u(0) \mathrm{e}^{-\frac{t}{\tau}}
$$

for all $t \in[0, T)$, then from Lemma 3, (13) is true for $\alpha>1$ and sufficiently small $\beta$ such that $\beta<\tau \alpha$. Since $H$ is bounded away from zero, then (13) is true for any $\alpha, \beta>0$.

From Lemma 1 and Lemma 4, we deduce the Corollary below.

Corollary 1. Let $\ell \geq 1$ and all other assumptions in Theorem 1, Lemma 2, Lemma 3, and Lemma 4 hold true. Define

$$
\begin{gathered}
g_{1}(A, H)=\frac{A^{p}}{H^{q}+b} \\
g_{2}(A, H)=\frac{A^{r}}{H^{s}}
\end{gathered}
$$

then there exist positive constant $C_{\ell}(T)$, such that

$$
\left\|g_{j}(A, H)\right\|_{L^{\ell}(\Omega)} \leq C_{\ell}(T), \quad j=1,2
$$

for all $0 \leq t<T$.

Proof. The proof to this Corollary follows from Lemma 3 and Lemma 4.

\section{Conclusion}

In this paper, we have studied the Gierer-Meinhardt system with Robin boundary conditions and Neumann boundary conditions on the activator and inhibitor respectively. Global existence of solutions have been obtained under the mixed boundary conditions using a priori estimates of solutions.

\section{References}

[1] Turing, A.M. (1952) The chemical basis of morphogenesis. Philosophical Transactions of the Royal Society of London Series B, 237, 37-72. https://doi.org/10.1098/rstb.1952.0012

[2] Meinhardt, H. (1982) Models of Biological Pattern Formation. Academic Press, London, New York.

[3] Meinhardt, H. (1998) The Algorithmic Beauty of Sea Shells. 2nd Edition, Springer, Berlin, Heidelberg. https://doi.org/10.1007/978-3-662-03617-4

[4] Gierer, A. and Meinhardt, H. (1972) A Theory of Biological Pattern Formation. Kybernetik(Berlin), 12, 30-39. https://doi.org/10.1007/BF00289234

[5] Masuda, K. and Takahashi, K. (1987) Reaction-Diffusion Systems in the Gierer-Meinhardt Theory of Biological Pattern Formation. Japan Journal of Applied Mathematics, 4, 47-58. https://doi.org/10.1007/BF03167754

[6] Maini, P.K., Wei, J. and Winter, M. (2007) Stability of Spikes in the Shadow Gierer-Meinhardt System with Robin Boundary Conditions. Chaos, 17, 037106. https://doi.org/10.1007/BF03167754

[7] Ni, W.-M., Suzuki, K. and Takagi, I. (2006) The Dynamics of a Kinetic Activator-Inhibitor Systems. The Journal of Differential Equations, 229, 426-465. https://doi.org/10.1016/j.jde.2006.03.011

[8] Dillon, R., Maini, P.K. and Othmer, H.G. (1994) Pattern Formation in Generalized 
Turing Systems. I. Steady-State Patterns in Systems with Mixed Boundary Conditions. The Journal of Mathematical Biology, 32, 345-393.

https://doi.org/10.1007/BF00160165

[9] Jiang, H. (2006) Global Existence of Solutions of an Activator-Inhibitor Systems. Discrete and Continuous Dynamical Systems, 14, 737-751.

https://doi.org/10.3934/dcds.2006.14.737

[10] Henry, D. (1981) Geometric Theory of Semilinear Parabolic Equations. SpringerVerlag, New York. https://doi.org/10.1007/BFb0089647

[11] Pazy, A. (1983) Semigroups of Linear Operators and Applications to Partial Differential Equations. Springer-Verlag, New York.

https://doi.org/10.1007/978-1-4612-5561-1

Submit or recommend next manuscript to SCIRP and we will provide best service for you:

Accepting pre-submission inquiries through Email, Facebook, LinkedIn, Twitter, etc. A wide selection of journals (inclusive of 9 subjects, more than 200 journals) Providing 24-hour high-quality service User-friendly online submission system Fair and swift peer-review system Efficient typesetting and proofreading procedure Display of the result of downloads and visits, as well as the number of cited articles Maximum dissemination of your research work

Submit your manuscript at: http://papersubmission.scirp.org/

Or contact am@scirp.org 